\title{
PAIN AND HEALTH-RELATED QUALITY OF LIFE AMONG OLDER PEOPLE WITH DIABETIC FOOT ULCER: A LITERATURE REVIEW
}

\author{
Aniawanis Makhtar ${ }^{*}$, Dinah Gould ${ }^{2}$ and Sally Anstey ${ }^{2}$ \\ ${ }^{1}$ Kulliyyah of Nursing, International Islamic University Malaysia, Malaysia \\ ${ }^{2}$ School of Healthcare Sciences, Cardiff University, Malaysia
}

\begin{abstract}
Little is known about how pain contributes to diabetic foot ulcer related health-related quality of life. The aim of this review was to explore the relationships between diabetic foot ulcer pain and health-related quality of life among older people. A literature review was conducted. MEDLINE (Ovid), the Cumulative Index of Nursing and the Allied Health Literature (CINAHL), and British Nursing Index (BNI) was carried out using MeSH terms diabetic foot ulcer, foot ulcer, pain, quality of life, health-related quality of life, and all studies published between 2000 and 2016 were considered in with both qualitative and quantitative approaches and in Malay and English. Thus, no restrictions were applied as regards study design. Twenty-seven studies were included; twenty- three used quantitative methods and four employed qualitative methods. No study focused only on older people. Pain was the factor most frequently identified as affecting health-related quality of life. It is, however, hard to draw any firm conclusions about older people's pain and health-related quality of life because of the lack of studies including older people. Thus, research about diabetic foot ulcer pain and health-related quality of life, especially focusing on the older people with diabetic foot ulcer, is needed.
\end{abstract}

Keywords: diabetic foot ulcer, pain, health-related quality of life, older people

\section{Introduction}

The world population is ageing as a result of increased life expectancy, lower fertility, and better health care services (United Nations 2013). Population ageing is happening in all regions of the world, with the older population in developing countries having a higher speed of growth compared to in developed countries (National Institute on Aging 2015). This obviously affects a society's economic and social structures, as well as its health care system. Ageing brings an increased risk of developing chronic non-communicable diseases (NCDs) such as hypertension, type 2 diabetes mellitus, stroke, and coronary heart disease (Wild et al. 2004). As a result, the health care burden in most nations will increase. Globally, at present, these chronic diseases are identified as being among the ten leading causes of death (World Health Organisation (WHO 2015)).

With the growing number of older people diagnosed with diabetes mellitus, Malaysia is not spared this phenomenon, as the prevalence of diabetes mellitus stands at $15.2 \%$ of the adult population (National Health and Morbidity Survey (NHMS 2011)). Two-thirds of the 177 million people with type 2 diabetes mellitus are estimated to live in the developing world ${ }^{6}$. The greatest increase in the prevalence of type 2 diabetes mellitus in older people is expected to occur in Asia and Africa, due to urbanisation and changes in lifestyle (Wild et al. 2004). Diabetes mellitus causes significant morbidity, disability, and mortality among older people (Gambert and Pinkstaff 2006). Its effects will increase health care costs to the patient, the community, and the nation (Hogan et al. 2003).

Diabetic foot ulcers are well known to be the most costly and devastating complication of diabetes mellitus due to the complexity of wound treatment and because they are a major reason for non-traumatic amputation (International Diabetes Federation 2013). The lifetime risk of a person with diabetes developing a foot ulcer could be as high as $25 \%$ (Singh 2005). As a result, it will cause substantial morbidity and impaired quality of life; it is the most important risk factor for lower-extremity amputation and results in high treatment costs and 
enormous economic losses (Margolis et al. 2005). As Malaysia's population is ageing and there is a rising prevalence of diabetes, it is expected that the number of diabetic foot ulcer patientswill also increase substantially.

Although there is a general misconception that pain or discomfort does not occur in diabetic foot ulcer patients, this is not entirely true for all patients, who despite having peripheral neuropathy may report severe and frequent pain (Baker 2012). Bradbury and Price (2011a) and Bradbury and Price (2011c [phase 1]) carried out a research to investigate the presence and characteristics of diabetic foot ulcer pain in 28 diabetic foot ulcer patients, and it was found that $86 \%(n=24)$ had diabetic foot ulcer pain. It occurs frequently and with high intensity despite peripheral neuropathy and in most cases, patients describe aching as the most common sensory pain. In addition, studies by Ribu et al. (2006) and Obilor and Adejumo (2014) suggested that specific ulcer pain from diabetic foot ulcers is more prevalent than expected and can affect the patient's quality of life. The aim of this review is to explore the relationships between diabetic foot ulcer pain and health-related quality of life among older people.

\section{Materials and Methods}

Electronic literature searches were conducted using the following databases to identify all published studies focusing on the relationships between diabetic foot ulcer pain and health-related quality of life: MEDLINE (Ovid), the Cumulative Index of Nursing and the Allied Health Literature (CINAHL), and British Nursing Index (BNI). To maximize the amount of literature, the review included studies published between 2000 and 2016 with no restriction on research design. Keywords that were used singularly and in combination were: diabetic foot ulcer, foot ulcer, pain, quality of life, health-related quality of life.

An article was included in the literature review if : i) the study concerning diabetic foot ulcers and health-related quality of life; ii) as this study focuses on adult populations, only studies that reported diabetic foot ulcers in adults aged $\geq 18$ years were included. Initially, only articles concerning patients aged 65 years and above were reviewed, but the database search had also captured articles with participants in younger age groups. For this reason, all studies that included participants aged 18 years and above with diabetic foot ulcers were included; iii) due to cost and time limitations for translating the articles from other languages into English or Malay, this review included only studies that were written in either language.

\section{Results}

In total 749 articles were found but only twenty-seven were found eligible for this review. The studies were conducted in various countries. Nine of the twenty-three papers were conducted in the United Kingdom and the others were conducted in Holland, the United States, Norway, France, Brazil, Spain, and Bermuda. Of the selected studies, only three were conducted in Asian countries: one in Iran, another in Taiwan, and another in Malaysia. Overall, it can be deduced that most of the studies were conducted within the western hemisphere including the United Kingdom, the United States, and several other European countries. Very few studies were found to have taken place in Asian settings. This shows the scarcity of information related to diabetic foot ulcer pain in South East Asia settings, including Malaysia.

The twenty-seven articles and reports were analyzed and the findings are discussed according to diabetic foot ulcer pain assessment, pain in diabetic foot ulcers, and diabetic foot ulcers, pain, and health-related quality of life.

\section{Diabetic foot ulcer pain assessment}

In the reviewed studies, three validated pain-rating scales were used by the diabetic foot ulcer patients to assess their pain status. The short-form McGill pain questionnaire (SF-MPQ) (Melzack 1987) was used in two of the 
studies (Bradbury and Price 2011a; Bradbury and Price 2011c [phase 1]) while the Leeds assessment of neuropathic symptoms and signs questionnaire (s-LANSS) was used in one (Yunus and Rajbhandari 2011). The visual analoque scale (VAS) was used in three (Bengstsson et al. 2008; Obilor and Adejumo 2014; Sibbald et al. 2013). However, in Ribu et al.'s (2006) study, pain was assessed by using two items from the physical health domain of the diabetic foot ulcer scale (DFS) (Abetz et al. 2002) and by asking the patients whether they had experienced any pain while walking or standing. In the study by Bradbury et al. (2011), pain was assessed using the following standardised local assessment criteria: the frequency of wound-related pain (none, intermittent (between dressing changes), at dressing change, continuous (between dressing changes) and the severity of wound-related pain (mild, moderate, severe, and non-evaluable). In the other studies, pain was assessed using a five-point Likert scale ("none," "mild," "moderate," "severe," and "extreme") (Sibbald et al. 2013), the domain of the American Academy of orthopaedic surgeons musculoskeletal outcomes measure (AAOS) (Evans and Pinzur 2005), and the domain of the medical outcomes study 36-item short-form health survey (SF-36).

The SF-MPQ was developed by Melzack (1987) to measure the different qualities of subjective pain experience. The questionnaire contains eleven sensory words and four affective words. The SF-MPQ also includes one item to present pain intensity and one item for a $100-\mathrm{mm}$ VAS for average pain. It was validated in patients with different types of pain.

The s-LANSS was developed by Bennet et al. (2005) to measure the symptoms and signs in neuropathic pain. The tool contains seven items and was validated in patients with different types of pain.

The VAS is a unidimensional measure of pain intensity that has been widely used in diverse adult populations (Hawker et al. 2011). It is one horizontal or vertical line $10 \mathrm{~cm}(100 \mathrm{~mm})$ in length. The respondent is told to anchor "no pain" as a 0 and "worst pain possible" as 10 (Burckhardt and Jones 2003).

In conclusion, only four validated measurements for pain were used in diabetic foot ulcer studies, such as the SF-MPQ, the s-LANSS, and the VAS. In Sibbald et al.'s (2013) study, however, they adopted a five-point Likert scale while Bradbury et al. (2011) used the standardised local assessment criteria for the measurement of pain. In other studies, a formal pain assessment was not used and instead, the researchers adopted domains from the DFS, the domain of the AAOS musculoskeletal outcomes measure, and the domain of SF-36. Therefore, the SFMPQ was found to be the most appropriate because the tool was designed to measure the sensory and affective aspects of pain and pain intensity.

\section{Pain in diabetic foot ulcer}

Diabetic foot ulcer pain was examined in six of the studies. A Norway diabetic foot ulcer pain prevalence study testified that $75 \%$ of patients had reported "some pain" in relation to diabetic foot ulcers (Ribu et al. 2006). Furthermore, $57 \%$ of the patients also reported ulcer pain while walking and/or standing and at night. This study also revealed that socio-demographic and clinical and ulcer characteristics were not significantly associated with any pain group. However, these findings are questionable because the sample was split into three groups: those who experienced pain none of the time, a little or some of the time, and most or all of the time. These groups were relatively small in size, which might affect the authors' ability to draw definitive conclusions about the differences in clinical characteristics among the three groups of patients. In relation to health-related quality of life, both the SF-36 and the DFS revealed significant differences between the three groups. Patients with the lowest scores in the physical and psychological domains of the questionnaire proved to have experienced pain either all the time or most of the time.

Similar findings were reported by Bengtsson et al. (2008), who investigated whether patients with neuropathic or neuroischaemic ulcers have experienced pain. The study revealed that $53 \%$ of the 101 patients reported having wound-related pain either intermittently or continuously. It was also reported that the presence of pain did not vary between aetiologies, with $34 \%$ of the patients with neuropathic ulcers reporting continuous pain 
compared to $30 \%$ of the neuro-ischaemic patients. The findings also concluded that the patients had encountered ulcer pain while walking, during dressing changes, and at night. The patients also described different feelings of pain, such as stinging, pricking, sore, burning, and pulsating/gripping.

A similar effect on pain was found in people with diabetic foot ulcers. Yunus and Rajbhandari (2011) surveyed the prevalence of neuropathic pain by ascertaining whether patients with and without diabetic foot ulcers had experienced the pain. In this study, $43.2 \%$ of the patients with diabetic foot ulcers had signs and symptoms of painful neuropathy, while only $18.2 \%$ had sought treatment. The authors noticed that the patients were often more concerned about their visible ulcer than about the invisible pain, and they appeared to ignore the symptoms of neuropathic pain ${ }^{18}$. The results showed that $43.2 \%$ of the participants with diabetic foot ulcers had symptoms and signs of painful neuropathy. The s-LANSS score was significantly higher in the diabetic foot ulcer group $(8.1 \pm 7.7$ versus $4.7 \pm 4.6 ; P=0.04)$ than in those without foot ulcers. However, there was no difference in the perception of pain in the 10 -point Likert scale $(3.9 \pm 3.6$ versus $3.3 \pm 3.0$; $P=$ not significant) between these two groups, which means that the patients with diabetic foot ulcers may have suffered from neuropathic pain but did not report it.

Another study describing the effects of pain found in people with diabetic foot ulcers was carried out by Bradbury and Price (2011a) and Bradbury and Price (2011c [phase 1]). Using the SF-MPQ (Melzack 1987), Bradbury and Price explored the presence and the characteristic of diabetic foot ulcer pain in 28 patients with diabetic foot ulcers at a specialist clinic. The study found that $86 \%(\mathrm{n}=24)$ of the patients had reported diabetic foot ulcer pain with a mean VAS score of 26.36 ( $\mathrm{SD}=24.29)$. Patients with neuro-ischaemic ulceration $(n=13)$ reported a higher mean score (mean $=32.2, \mathrm{SD}=24.6)$ compared to patients with neuropathic ulceration $(\mathrm{mean}=$ 21.6, $\mathrm{SD}=24.6)$. Using the $\mathrm{SF}-\mathrm{MPQ}$, the authors found the patients were more likely to use five descriptors: aching, hot-burning, tender, sharp, and tiring/exhausting. These painful sensations indicate a combination of nociceptive (aching, tender) and neuropathic (hot-burning, sharp) pain.

In a recent study by Obilor and Adejumo (2014), pain was examined in 14 patients with diabetic foot ulcers. Their finding showed that all the participants experienced diabetic foot ulcer-related pain either at rest $(n=4)$, during the performance of activity daily living (ADL) particularly in their movement $(n=8)$, or at night $(n=5)$. Measuring pain intensity on a VAS of $0-10 \mathrm{~cm}$, the majority of the patients $(85.7 \%, \mathrm{n}=12)$ reported the experience to be of moderate to severe pain intensity, with a mean of 5.43 (SD 2.24, range 2-10). The patients frequently chose words such as stinging, tingling, sharp, stabbing, throbbing, and aching. These painful sensations indicate a combination of nociceptive (sharp, stabbing, throbbing, aching) and neuropathic (stinging, tingling) pain. In terms of health-related quality of life, the diabetic foot ulcer-related pain experienced by the patients was found to be significantly related to the physical functioning, social functioning, and general mental health domains of the medical outcomes survey short-form 12 (SF-12), with a majority of the patients having lower scores in both physical and psychological domains.

In conclusion, only six studies were found to have focused specifically on individuals with diabetic foot ulcer pain. Nevertheless, all the studies highlighted the need for further research to examine diabetic foot ulcer pain.

\section{Diabetic foot ulcers, pain, and health-related quality of life}

A large and growing body of literature has documented the health-related quality of life of patients with diabetic foot ulcers, but studies that focus on the pain experience of people with diabetic foot ulcers are limited in number. In the reviewed studies, pain is often discussed in terms of the relationship between diabetic foot ulcers and health-related quality of life. One example is the study by Nabuurs-Franssen et al. (2005), who from the SF36 indicated that those patients with persisting ulcers had lower health-related quality of life scores than those with healed ulcers, particularly in physical functioning. The findings of the study also revealed that compared to persistent ulcer patients, patients with healed ulcers had complained of pain. 
The above findings supported the discovery by Ribu et al. (2007) in relation to the effects of quality of life of people with diabetic foot ulcers. The results showed that the diabetic foot ulcer group had significantly lower scores in all the SF-36 subscales compared to both the diabetic and nondiabetic general population, particularly in areas concerned with physical health and performing ADL. The authors also acknowledged that compared to diabetic patients, patients with diabetic foot ulcers had complained about having more pain, but did not explain the cause of their pain.

Similar to previous studies conducted in the European countries, patients with diabetic foot ulcers in Asia are also affected in terms of physical functioning and mental health. A study by Huang et al. (2012) on 131 consecutive patients who attended a diabetic clinic in Shanghai revealed that the scores in the subscale of physical functioning were low in diabetic foot ulcer patients. The study also statistically attested the significance of bodily pain among patients with diabetic foot ulcers. In another study, Boutoille et al. (2008) confirmed that having a diabetic food ulcer has a great influence on patients' quality of life especially in the bodily pain domain. Nonetheless, the discussion was limited to the relation of foot ulcers to peripheral arterial disease.

A similar condition was observed in Malaysia. One example is a local study conducted by Mazlina et al. (2011) on 140 diabetic patients with foot problems and 134 diabetic patients without foot problems at an outpatient diabetic foot clinic in University Malays Medical Centre, Malaysia. The result from all the eight SF-36 domains indicated that patients with foot problems had lower health-related quality of life scores than those without foot problems, In particular, restrictions in physical functioning limited the patients' abilities to perform daily living activities, and they also had bodily pain. This study supported the necessity for further exploration of the issue of pain and diabetic foot ulcers.

A large quantitative cross-sectional study was conducted by Siersma et al. (2013) across ten European countries participating in the Eurodiale study. In the study, the patients reported a poor overall health-related quality of life, with problems primarily in the mobility and pain/discomfort domains. Among the comorbidities, the inability to stand or walk without help was the most important determinant of a decreased health-related quality of life in all five domains of the Euro-Qol-5D. The authors suggested that a clinical diagnosis of infection, peripheral arterial disease, and polyneuropathy were associated with the pain/discomfort domain.

Other studies have attested that pain is not a significant factor among diabetic foot ulcer patients (de Meneses et al. 2011; Goodridge et al. 2006; Meijer et al. 2001; Evans and Pinzur 2005; Willrich et al. 2005). For example, in a study by Meijer et al. (2001), the results from the research and development 36-item form (RAND-36) and the walking and walking stairs questionnaire (WWSQ) indicated lower scores for patients with diabetic foot ulcers than for those without diabetic foot ulcer. The researchers also concluded that even a clinically stable diabetic foot ulcer may cause significant impairment in the quality of life, particularly in the domains of physical functioning, social functioning, physical role, and health experience. However, there was no mention of the relationship between having a diabetic foot ulcer and pain. This contradicts the findings of other studies, which suggested pain was a significant factor. This study did not support the hypothesis that pain from diabetic foot ulcers is prevalent and has a significant influence on a patient's quality of life.

In Goodridge et al.'s (2006) cross-sectional study, they conducted a descriptive study to compare the quality of life between patients with active and patients with healed foot ulcers. The findings showed the unhealed diabetic foot ulcer group reported significant limitations on their activities, work, and social life, even if the diabetic foot ulcer had a low Wagner classification grade. There was little difference between the two groups with regard to the effect of pain on their activity; $40 \%$ of the participants in each group did not consider pain to be a problem. In addition, no difference was noted in the mental health domain between the two groups. Similar to the study by Meijer et al. (2001), this study also did not support the hypothesis that pain from diabetic foot ulcers is prevalent and has a significant influence on patients' quality of life. However, the study adds to the body of 
knowledge on this area while supporting other findings on the negative impacts of diabetic foot ulcers on quality of life.

In a quantitative study, Evans and Pinzur (2005) measured the impacts of foot ulcers on the physical, mental, emotional, and social aspects of patients' lives. The results revealed a statistically significant difference in the physical functioning of the patients. Although pain was not a significant component in this study, the diabetic foot ulcer patients complained of extreme pain during resting, walking on an uneven surface, and stair climbing. The results of the study will be a platform for further investigation on diabetic foot ulcer pain and health-related quality of life.

In qualitative studies, pain is a dominant theme and is consistent across all studies (Ribu and Wahl 2004; Ashford et al. 2000; Watson-Miller 2006; Bradbury and Price 2011b; Bradbury and Price 2011c [phase 2]). In these studies, pain was significant; it was described as the worst symptom and had a profound effect on the quality of life of the patients. Ribu and Wahl (2004) conducted a qualitative study to understand the patients' perspective of living with diabetic foot ulcers. The data analysis revealed six categories of problems commonly experienced in relation to living with diabetic foot ulcers, one of which concerned pain. The study found that almost all the patients experienced pain at some time. Although one patient discussed it in relation to an active Charcot arthropathy, others reported how pain from the ulcer had woken them up at night and how they had to recline in certain positions to avoid any pressure on the ulcer, which would cause pain. The patients also claimed that pain was felt while walking even for short distances. The study also highlighted the effects of pain in causing sleep deprivation and fatigue, which affected the overall quality of life. In the study, three patients avoided taking analgesia despite experiencing pain due to a fear of analgesia dependency.

In a similar study using a phenomenological approach, Ashford et al. (2006) conducted research to address the quality of life issues among patients with diabetic foot ulcers. The results suggested that the patients suffered emotionally, financially, physically, and socially due to diabetic foot ulcers. The data analysis also revealed six categories of problems commonly experienced in relation to living with diabetic foot ulcers, one of which concerned pain. Half of the patients (50\%) reported pain arising from the ulcer. Pain was also reported during dressing change and while lying down. The ulcer pain had also caused the patients difficulty in walking and resulted in reduced mobility. The study was successful in its aims to allow the diabetic foot ulcer patients to express their thoughts on issues regarding their quality of life and diabetes. Accordingly, the authors suggested that it is crucial for healthcare providers to use a holistic approach when dealing with patients with diabetes.

Watson-Miller (2006) investigated the experiences of six patients of living with diabetic foot ulcers. The participants shared their experiences and reported that the pain caused by diabetic foot ulcers had had negative effects on their quality of life. Although Watson-Miller (2006) did not investigate this aspect further, the author highlighted the importance of making a holistic assessment of people's quality of life. The author also emphasised the need for clinicians to be aware of the difficulties of people with diabetic foot ulceration. Interestingly, the patients in this study did not report any social isolation. However, it should be noted that this study had a very small number of participants and that the results are not comparable to the findings reported in other studies, which did note the problem of social isolation (Ribu and Wahl, 2004; Bradbury and Price 2011b).

The most recent qualitative study on the health-related quality of life of patients with diabetic foot ulcers was undertaken using face-to-face semi-structured interviews with three diabetic foot ulcer patients from a specialist outpatient diabetic foot clinic (Bradbury and Price 2011b; Bradbury and Price 2011c [phase 2]). Four major themes emerged linking pain and quality of life: the experience of pain; the physical effects of pain; coping, support and social impact and the psychological impact. The study found that as a result of diabetic foot ulcer pain, the patients were affected physically and psychologically particularly with regard to mobility, sleep, and social life. Pain was also reported during dressing changes and while lying down. The ulcer pain also caused the patients difficulty in walking. The study also identified the diabetic foot ulcer patients' feelings of depression, isolation, and loss of independence, which appeared to be the consequences of ulcer pain. The strength of this 
study is that it identified a significant gap in the research; for those diabetic foot ulcer patients who experienced pain, the pain, in turn, had negative impacts on their quality of life. Further qualitative research was recommended into the patients' lived experiences of diabetic foot ulcer pain in order to help healthcare professionals understand the relevance of holistic diabetic foot care and service provision.

Other studies have revealed little about pain and its impacts; as their purpose was not focused on pain specifically (Willrich et al. 2005; Winkley et al. 2009; Jelsness-Jorgensen et al. 2011; de Meneses et al. 2011; Morales et al. 2011; Sanjari et al. 2011).

\section{Discussion}

In summary, the literature review established that diabetic foot ulcer pain significantly affects the physical, social, and the psychological aspects of health-related quality of life. Pain was identified as a major concern, with reports often ignored.

The major drawback of this review is that no study specifically focused on older people. Though the studies had varying representations of this group, they were mostly carried out with a small sample size. This means that no conclusions about pain and health-related quality of life can be drawn for this age group. Thus, this can be considered an important area for further research.

Another issue identified from the literature review was the different scales or instruments used in the studies. These scales probably measured different facets of the phenomena and therefore cannot be interpreted interchangeably. For example, although Ribu et al. (2006) actually acknowledged that diabetic foot ulcer pain was important, they did not perform any formal assessments on the patients because the study primarily aimed to evaluate the specifics of diabetic foot ulcer pain. This raised the need of ascertaining which questionnaires can best measure pain and its relationships with health-related quality of life.

In terms of research methods, the most common limitations of the quantitative studies were the small sample size, the lack of reporting of a sample size calculation, and the inexplicit inclusion and exclusion criteria. For instance, in the study by Bradbury and Price (2011a) and Bradbury and Price (2011c [phase 1]), limited inferential statistic was applied due to the small sample size. Similarly, in the study by Bengtsson et al. (2008), no inferential statistic was applied to provide more detailed information on the relationship between pain sensation and other variables, such as patient demographic data and wound characteristics.

The review indicates that a large number of studies pertaining to this issue have been carried out in the United States and the European countries. However, very little research has been conducted in Asia, with only one study relating to health-related quality of life and diabetic foot ulcers being conducted in Malaysia. The study by Mazlina et al. (2010), however, did not evaluate the degree of pain associated with diabetic foot ulcers. Their findings nevertheless suggested that the experience of pain from diabetic foot ulcers has a negative impact on the physical as well as the mental health of patients, and thus, further exploration is required in this area.

\section{Conclusion}

The conclusion derived from the study is that the reduced quality of life among diabetic patients can be attributed to the pain experienced by them. Despite a number of studies having looked into the relationship between health-related quality of life and diabetic foot ulcers, none has included a detailed discussion on the effects of pain, specifically focused on older people. This objective appears not to be the primary aim of most of these studies; thus, their findings may not be used to draw substantial conclusions on the subject. However, the discoveries can provide scholars with a background of the subject and pave the way for a detailed study on the nature and degree of pain. This paper highlights the implication of literature review for a future study on the relationships between diabetic foot ulcer pain and health-related quality of life among older people in Malaysia. 


\section{Acknowledgment}

The authors would like to express a deepest gratitude to Ministry of Education, Malaysia, and International Islamic University for the funding and supports.

\section{References}

Abetz, L. et al. The diabetic foot ulcer scale (DFS): a quality of life instrument for use in clinical trials. Practical Diabetes International. 2002. 19(6), pp. 167-175.

Ashford, R.L. et al. Perception of quality of life by patients with DFUs. Diabetic Foot. 2000; 3(4), pp. 150-155.

Baker, N. Implications of dressing-related trauma and pain in patients with diabetes. Diabetic Foot. 2012; J 15, pp. 1-8.

Bengtsson. L. et al. Wound-related pain is underestimated in patients with diabetic foot ulcers. J Wound Care. 2008; 17(10), pp. 43.

Bennet, M. I. et al. The s-LANSS score for identifying pain of predominantly neuropathic origin: validation for use in clinical and postal research. J Pain. 2005; 6, pp. 149-158.

Boutoille, D. et al. Quality of life with diabetes associated foot complications: comparison between lower-limb amputation and chronic foot ulceration. Foot Ankle Int 29. 2008; pp. 1074-1078.

Bradbury, S. and Price, P. Diabetic foot ulcer pain: the hidden burden (part two). EWMA Journal. 2011 b; 11 (2), pp. 25-37.

Bradbury, S. and Price, P. Diabetic foot ulcer pain: the hidden burden (part one). EWMA Journal. $2011 \mathrm{a} ; 11$ (2), pp. 25-37.

Bradbury, S. and Price, P. The impact of diabetic foot ulcer pain on quality of life. Wounds UK. 2011c; 7 (4), pp. 32-49.

Bradbury, S. et al. Case series evaluation of a silver non-adherent dressing. Wounds UK Journal. 2011; 7(2), pp.12-19.

Burckhardt, C. S. and Jones, K. D. Adult measures of pain: The Mcgill pain questionnaire (MPQ), rheumatoid arthiritis pain scale, SF-MPQ, verbal description scale (VDS), VAS and West Haven-Yale Multi Disiplinary Pain Inventory (WHYMP). Arthritis Care and Research. 2003; 49 (5), pp. 96-104.

de Meneses, L. C. et al. Health related quality of life and self-esteem in patients with diabetic foot ulcers: results of a cross-sectional comparative study. Ostomy Wound Management. 2011; 57 (3), pp. 36-43.

Evans, A. R. and Pinzur, M. S. Health -related quality of life of patients with diabetes and foot ulcers. Foot and Ankle International. 2005; 26 (1), pp. 32-37.

Gambert, S. and Pinkstaff, S. Emerging epidemic: diabetes in older adults: demography, economic impact, and pathophysiology. Diabetes Spectrum. 2006;19(4), pp. 221-228.

Goodridge, D. et al. Quality of life of adults with unhealed and healed DFUs. Foot and Ankle International. 2006; 27 (4), pp. 274-280.

Hawker, G. et al. Measures of adult pain: Visual analog scale for pain (VAS Pain), numeric rating scale for pain (NRS Pain), McGill pain questionnaire (MPQ), short-form McGill pain questionnaire (SF-MPQ), chronic pain grade scale (CPGS), short form-36 bodily pain scale. Arthritis Care Res. 2011; 63 (S11), pp. S240-S252.

Hogan, P. et al. Economic costs of diabetes in the US in 2002. Diabetes Care. 2003; 26(3), pp. 917-932.

Huang, Y. et al. The investigation of demographic characteristics and the health related quality of life in patients with diabetic foot ulcers at first presentation. International Journal of Lower Extremity Wound. 2012; 11(3), pp. 187-193.

International Diabetes Federation. Diabetes Atlas. $6^{\text {th }}$ ed. International Diabetes Federation. [Online]. Available from: http://www.idf.org/sites/default/files/EN_6E_Atlas_Full_0.pdf [Accessed: 22 October 2015].

Jelsness-Jørgensen, L. P. et al. Measuring health related quality of life in non-complicated diabetes patients may be an effective parameter to assess patients at risk of a more serious course: a cross- sectional study of two diabetes outpatient groups. Journal of Clinical Nursing. 2011; 20, pp. 1255-1263. 
Margolis, D. et al. Diabetic neuropathic foot ulcers and amputation. Wound Repair and Regeneration. 2005; 13(3), pp. 230-236.

Mazlina, M. et al. Health related quality of life in patients with diabetic foot problems in Malaysia. Med J Malaysia. 2011; 66 (3), pp. 234- 238.

Meijer, J. W. G. et al. Quality of life in patients with diabetic foot ulcer. Disability and Rehabilitation. 2001; 23(8), pp. 336-340.

Melzack, R. The short-form McGill Pain Questionnaire. Pain. 1987; 30(2), pp. 191.

Morales, E. G. et al. Impact of diabetic foot related complications on the health related quality of Life (HRQol) of patients a regional study in Spain. International Journal of Lower Extremity Wounds 2011; 10 (1), pp. 6-11.

Nabuurs-Franssen, M.H. et al. Health related quality of life of diabetic foot ulcer patients and their caregivers. Dialectologies. 2005. 48, pp.1906-1910.

National Health and Morbidity Survey Malaysia. National health and morbidity survey Malaysia 2011 fact sheet [Online]. Available at: www.moh.gov.my/images/.../NHMS\%202011\%20FACT\%20SHEET.pdf [Assessed: 20 August 2013].

National Institute on Aging. Global health and aging [Online]. Available at:https://www.nia.nih.gov/research/publication/...aging/humanitys-aging [Accessed: 10 September 2015].

Obilor, H. N. and Adejumo, P. O. Assesment of diabetic foot ulcer- related pain and its relationship to quality of life. Wound Practice and Research. 2014; 23 (3), pp. 123-131.

Ribu, L. and Wahl, A. Living with diabetic foot ulcer: a life of fear, restriction and pain. Ostomy/Wound Management 2004; 50 (2), pp. 57-67.

Ribu, L. et al. A comparison of the health related quality of life in patients with diabetic foot ulcers with a diabetes group and a nondiabetes group from the general population. Quality of Life Research. 2007; 16, pp. 179-189.

Ribu, L. et al. The prevalence and occurance of diabetic foot ulcer pain and its impact on the health related quality of life. The Journal of Pain. 2006; 7 (4), pp. 290-299.

Sanjari, M. et al. A cross- sectional study in Kerman, on the effect of diabetic foot ulcer on health related quality of life. International Journal of Lower Extremity Wounds. 2011; 10(4), pp. 200-206.

Sibbald, R. G. et al. Pain in diabetic foot ulcers. Ostomy/Wound Management. 2003; 49 (4A Suppl), pp. 24-29.

Siersma, V. et al. Importance of factors determining the low health related quality of life in people presenting with a diabetic foot ulcer: the Eurodiale study. Diabet Med. 2013; 30, pp.1382-1387.

Singh, N. et al. Preventing foot ulcers in patients with diabetes. Journal of the American Medical Association 2005; 293, pp. 217-228.

[United Nations. World population ageing [Online] Available at: http://www.un.org/en/development/desa/population/publications/pdf/ageing/WorldPopulationAgeing2013.pdf [Accessed: 10 September 2015].

Watson-Miller, S. Living with a diabetic foot ulcer: A phenomenological study. Journal of Clinical Nursing. 2006; 15, pp. 1336-1337.

Wild S, et al. Global prevalence of diabetes: estimates for the year 2000 and projections for 2030. Diabetes Care. 2004; 27(5), pp. $1047-1053$.

Wilirich, A. et al. Health- related quality of life, cognitive function, and depression in diabetic patients with foot ulcer or amputation, a preliminary study. Foot \& Ankle International. 2005; 26(2), pp. 128-134.

Winkley, K. et al. Quality of life in people with their first diabetic foot ulcer: A prospective cohort study. Journal of the American Podiatric Medical Association. 2009; 99(5), pp 406-414.

World Health Organization. The top 10 causes death [Online]. Avalilable at: http://www.who.int/mediacentre/factsheets/fs310/en/ [Assessed: 10 May 2015].

Yunus, Y. M. and Rajbhandari, S. M. Insenate foot of diabetic foot ulcer can have underlying silent neuropathic pain. Int Wound J. 2011; 8, pp. 301-305. 